\title{
Optimal Colorings with Rainbow Paths
}

\author{
O. Bendele and D. Rautenbach \\ Institute of Optimization and Operations Research, Ulm University, Ulm, Germany \\ oliver.bendele@uni-ulm.de, dieter.rautenbach@uni-ulm.de
}

\begin{abstract}
Let $G$ be a connected graph of chromatic number $k$. For a $k$-coloring $f$ of $G$, a full $f$-rainbow path is a path of order $k$ in $G$ whose vertices are all colored differently by $f$.

We show that $G$ has a $k$-coloring $f$ such that every vertex of $G$ lies on a full $f$-rainbow path, which provides a positive answer to a question posed by Lin (Simple proofs of results on paths representing all colors in proper vertex-colorings, Graphs Combin. 23 (2007) 201-203). Furthermore, we show that if $G$ has a cycle of length $k$, then $G$ has a $k$-coloring $f$ such that, for every vertex $u$ of $G$, some full $f$-rainbow path begins at $u$, which solves a problem posed by Bessy and Bousquet (Colorful paths for 3-chromatic graphs, arXiv 1503.00965v1). Finally, we establish some more results on the existence of optimal colorings with (directed) full rainbow paths.
\end{abstract}

Keywords: chromatic number; circular chromatic number, rainbow path MSC2010: 05C15, 05C38

\section{Introduction}

Let $G$ be a finite, simple, and undirected graph with vertex set $V(G)$. For a positive integer $k$, let $[k]$ be the set of all positive integers at most $k$. A $k$-coloring of $G$ is a function $f: V(G) \rightarrow[k]$ such that $f(u) \neq f(v)$ for every two adjacent vertices $u$ and $v$ of $G$. The chromatic number $\chi(G)$ of $G$ is the minimum $k$ such that $G$ has a $k$-coloring. For positive integers $n$ and $d$ with $n \geq 2 d$ and $\operatorname{gcd}(n, d)=1$, an $(n, d)$-coloring of $G$ is a function $c: V(G) \rightarrow[n]$ such that $d \leq|c(u)-c(v)| \leq n-d$ for every two adjacent vertices $u$ and $v$ of $G$. The circular chromatic number $\chi_{c}(G)$ of $G$ is the infimum of $\frac{n}{d}$ over all $(n, d)$-colorings of $G$. It is well-known [8, 9] that this infimum is a minimum, and that $\chi(G)-1<\chi_{c}(G) \leq \chi(G)$, which implies $\left\lceil\chi_{c}(G)\right\rceil=\chi(G)$.

If $f$ is a $k$-coloring of $G$, then an $f$-rainbow path is a path $P$ in $G$ such that $f(u) \neq f(v)$ for every two distinct vertices $u$ and $v$ of $P$. The path $P$ is full if it has order $k$, that is, if all $k$ colors appear on $P$. For a positive integer $k$ at least 3 , let $C_{k}$ denote the cycle of order $k$.

With the following result we give an affirmative answer to a problem posed by Lin [6].

Theorem 1 For every connected graph $G$ of chromatic number $k$, there is a k-coloring $f$ of $G$ such that every vertex of $G$ lies on a full $f$-rainbow path.

In [1] Akbari, Khaghanpoor, and Moazzeni conjectured that every connected graph $G$ of chromatic number $k$ that is distinct from $C_{7}$, has a $k$-coloring $f$ such that, for every vertex $u$ of $G$, some full $f$-rainbow path begins at $u$. Alishahi, Taherkhani, and Thomassen [3] showed the existence of a $k$-coloring $f$ such that, for every vertex $u$ of $G$, some $f$-rainbow path of order $\left\lfloor\chi_{c}(G)\right\rfloor$ begins at $u$, which implies the conjecture whenever $\chi_{c}(G)=\chi(G)$. A close look at the proofs in [3] actually yields the following. 
Theorem 2 Let $k, n$, and $d$ be positive integers with $n \geq 2 d, \operatorname{gcd}(n, d)=1$, and $\frac{n}{d}<k$. If $G$ is a connected graph with $\chi(G)=k$ and $\chi_{c}(G)=\frac{n}{d}$, then $G$ has a $k$-coloring $f$ such that

- for at least $\left(\frac{\chi(G)\left(\chi_{c}(G)+1-\chi(G)\right)}{\chi_{c}(G)}\right)|V(G)|$ vertices $u$ of $G$, some full $f$-rainbow path begins at $u$, and

- for the remaining vertices $v$ of $G$, some $f$-rainbow path of order $k-1$ begins at $v$.

Akbari, Liaghat, and Nikzad [2] proved the conjecture of Akbari, Khaghanpoor, and Moazzeni for graphs of chromatic number $k$ that contain a clique of order $k$. Bessy and Bousquet [4] proved the conjecture for $k=3$. Furthermore, they verified it for $k=4$ provided that $G$ contains a cycle of length 4 , and asked whether it holds for graphs that contain a cycle of length $k$ (cf. Problem 10 in [4]). We answer this question and provide some related results.

Theorem 3 Let $G$ be a connected graph of chromatic number $k$. If $G$ contains a cycle of length $k$, then $G$ has a $k$-coloring $f$ such that, for every vertex $u$ of $G$, some full $f$-rainbow path begins at $u$.

Let $G$ be a graph of chromatic number $k$, and let $D$ be an orientation of $G$. Gallai [5] and Roy [7] showed that $D$ contains a directed path of order $k$. As a possible strengthening of this result, Lin [6] asked whether $G$ has a $k$-coloring $f$ such that $D$ contains a directed full $f$-rainbow path. For $k \leq 2$, this is trivial. We show the existence of such an $f$ for $k=3$.

Theorem 4 For every orientation $D$ of a graph $G$ of chromatic number 3 , there is a 3-coloring $f$ of $G$ such that $D$ contains a directed full $f$-rainbow path.

All proofs are postponed to the next section.

\section{Proofs}

For positive integers $a, b$, and $d$, let $a \bmod d$ denote the residue of $a$ modulo $d$, and let $a \equiv_{d} b$ abbreviate $(a-b) \bmod d=0$.

An essential tool from [3] is the following result.

Theorem 5 (Alishahi, Taherkhani, and Thomassen [3]) Let $n$ and $d$ be positive integers with $n \geq 2 d$ and $\operatorname{gcd}(n, d)=1$. If $G$ is a connected graph with $\chi_{c}(G)=\frac{n}{d}$, then $G$ has an $(n, d)$-coloring c such that, for every vertex $u$ of $G$, there is a path $u_{1} \ldots u_{n}$ in $G$ with $u_{1}=u$ and $c\left(u_{i+1}\right) \equiv_{n} c\left(u_{i}\right)+d$ for every $i \in[n-1]$.

Proof of Theorem 1: Let $G$ be a connected graph of chromatic number $k$. In view of the mentioned results from [3], we may assume that $G$ has circular chromatic number $\frac{n}{d}$ for positive integers $n$ and $d$ with $n \geq 2 d$ and $\operatorname{gcd}(n, d)=1$ such that $\frac{n}{d}<k$. Note that this implies $d \geq 2$. Let $c$ be an $(n, d)$-coloring of $G$ as in Theorem 5. Since $c$ is an $(n, d)$-coloring and $k=\left\lceil\frac{n}{d}\right\rceil$, the function $f: V(G) \rightarrow[k]: u \mapsto\left\lceil\frac{c(u)}{d}\right\rceil$ is a $k$-coloring of $G$.

Let $u$ be a vertex of $G$. If $v$ is a vertex with $c(v) \equiv_{n} c(u)+d$, then the definition of $f$ implies

$$
f(v)=\left\{\begin{aligned}
f(u)+1, & f(u) \leq k-2 \\
k, & f(u)=k-1 \text { and } c(u) \bmod d \in[n \bmod d], \\
1, & f(u)=k-1 \text { and } c(u) \bmod d \notin[n \bmod d], \text { and } \\
1, & f(u)=k .
\end{aligned}\right.
$$


By Theorem 5, $G$ contains a path

$$
u_{1} \ldots u_{k} \text { such that } u_{1}=u \text { and } c\left(u_{i+1}\right) \equiv_{n} c\left(u_{i}\right)+d \text { for every } i \in[k-1] \text {. }
$$

By (11), we obtain that if $c(u) \bmod d \in[n \bmod d]$, then $u_{1} \ldots u_{k}$ is a full $f$-rainbow path, and if $c(u) \bmod d \notin[n \bmod d]$, then $u_{1} \ldots u_{k-1}$ is an $f$-rainbow path of order $k-1, f\left(u_{1}\right)=f\left(u_{k}\right)$, and $k \notin\left\{f\left(u_{1}\right), \ldots, f\left(u_{k}\right)\right\}$.

For $i \in[n]$, let $V_{i}=\{u \in V(G): c(u)=((i-1) d \bmod n)+1\}$. Since $\operatorname{gcd}(n, d)=1, V_{1} \cup \ldots \cup V_{n}$ is a partition of $V(G)$.

Let $f_{0}=f$. We will now define $k$-colorings $f_{1}, \ldots, f_{n}$ of $G$ such that $f_{0}=f$, and for every integer $i$ with $0 \leq i \leq n-1$,

(i) $f_{i+1}$ and $f_{i}$ coincide on $V(G) \backslash V_{i+1}$, and

(ii) every vertex in $V_{1} \cup \cdots \cup V_{i+1}$ lies on a full $f_{i+1}$-rainbow path.

Note that $f_{n}$ will be a $k$-coloring of $G$ such that every vertex of $G$ lies on a full $f_{n}$-rainbow path.

Since $k=\left\lceil\frac{n}{d}\right\rceil>\frac{n}{d}$, we obtain $c(u)=(i-1) d+1$ for $u \in V_{i}$ with $i \in[k]$, which implies $c(u) \bmod d=1 \in[n \bmod d]$. Hence, every vertex in $V_{1} \cup \cdots \cup V_{k}$ lies on a full $f$-rainbow path, and we may define $f_{1}, \ldots, f_{k}$ all to be equal to $f$.

Now, for some integer $i$ with $k \leq i<n$, we may assume that $f_{i}$ has already been defined. By (i), the functions $f_{i}$ and $f$ coincide on $V_{j}$ for every $j$ with $i+1 \leq j \leq n$. Since $f_{1}=\ldots=$ $f_{k}=f$, the functions $f_{i}$ and $f$ coincide on $V_{j}$ for every $j$ with $1 \leq j \leq k$. Altogether, the functions $f_{i}$ and $f$ coincide on $V_{i+1} \cup \ldots \cup V_{i+k}$, where we identify indices modulo $n$. Therefore, if $(((i+1)-1) d \bmod n)+1) \bmod d \in[n \bmod d]$, then for every vertex $u$ in $V_{i+1}$, a path $u_{1} \ldots u_{k}$ as in (2) is a full $f_{i}$-rainbow path, that is, every vertex in $V_{i+1}$ lies on a full $f_{i}$-rainbow path, and we may define $f_{i+1}=f_{i}$. Hence, we may assume that $\left.(((i+1)-1) d \bmod n)+1\right) \bmod d \notin[n \bmod d]$.

Let $u$ be a vertex in $V_{i+1}$. Let $u_{1} \ldots u_{k}$ be a path as in (2). Since $f_{i}$ and $f$ coincide on $V_{i+1} \cup \ldots V_{i+k}$, the path $u_{1} \ldots u_{k-1}$ is an $f_{i}$-rainbow path of order $k-1, f_{i}\left(u_{1}\right)=f_{i}\left(u_{k}\right)$, and $k \notin\left\{f_{i}\left(u_{1}\right), \ldots, f_{i}\left(u_{k}\right)\right\}$. If $u$ has a neighbor $v$ with $f_{i}(v)=k$, then $v u_{1} \ldots u_{k-1}$ is a full $f_{i}$-rainbow path that contains $u$. Therefore, if $X_{i+1}$ is the set of vertices in $V_{i+1}$ that do not lie on a full $f_{i}$-rainbow path, then no vertex in $X_{i+1}$ has a neighbor $v$ with $f_{i}(v)=k$, and the function $f_{i+1}$ with

$$
f_{i+1}(x)=\left\{\begin{aligned}
f_{i}(x), & x \in V(G) \backslash X_{i+1}, \text { and } \\
k, & x \in X_{i+1}
\end{aligned}\right.
$$

is a $k$-coloring of $G$ that satisfies (i). Since we only changed $f_{i}$ on vertices that do not lie on a full $f_{i}$-rainbow path, every vertex of $G$ that lies on a full $f_{i}$-rainbow path also lies on a full $f_{i+1}$-rainbow path. Furthermore, if $u$ is in $X_{i+1}$, then a path as in (2) is a full $f_{i+1}$-rainbow path that starts in $u$. Altogether, (ii) holds, which completes the proof.

Proof of Theorem 2: Let $k, n, d$, and $G$ be as in the statement of the theorem, that is, in particular, $k=\left\lceil\frac{n}{d}\right\rceil$. Let $c$ be an $(n, d)$-coloring of $G$ as in Theorem 5. Let $I=\{i \in[n]: i \bmod d \in[n \bmod d]\}$. Since $k=\left\lceil\frac{n}{d}\right\rceil$, we have $|I|=k(n \bmod d)$ and $n=(k-1) d+n \bmod d$, which implies $\frac{|I|}{n}=$ $\frac{k}{n}(n-(k-1) d)=\frac{\chi(G)}{\chi_{c}(G)}\left(\chi_{c}(G)+1-\chi(G)\right)$.

Note that $c^{\prime}: V(G) \rightarrow[n]$ with

$$
c^{\prime}(x)=\left\{\begin{aligned}
c(x)+1, & c(x) \leq n-1 \text { and } \\
1, & c(x)=n
\end{aligned}\right.
$$


is an $(n, d)$-coloring of $G$ for which paths as in Theorem 5 (with $c$ replaced by $c^{\prime}$ ) still exist. Iteratively applying this shifting operation, we may assume that $\frac{\left|c^{-1}(I)\right|}{|V(G)|} \geq \frac{|I|}{n}$. Now, Theorem 2 is a consequence of the observations following (1) and (2) in the proof of Theorem 11,

Proof of Theorem 3]: Our proof relies on arguments from [2, 4].

Let $G$ be a connected graph of chromatic number $k$. For a $k$-coloring $f: V(G) \rightarrow[k]$ of $G$, let $D_{f}$ be the digraph with vertex set $V(G)$ and arc set

$$
\left\{(u, v): u v \in E(G) \text { and } f(v) \equiv_{k} f(u)+1\right\} .
$$

As observed in [4], the conjecture of Akbari, Khaghanpoor, and Moazzeni [1] holds for $G$ if $D_{f}$ contains a directed cycle. Therefore, we may assume that

$$
D_{f} \text { is an acyclic digraph for every } k \text {-coloring } f \text { of } G \text {. }
$$

Let $X$ be a set of vertices of $G$. Let $V_{f}^{+}(X)$ be the set of vertices $y$ of $G$ such that $D_{f}$ contains a directed path from a vertex $x$ in $X$ to $y$. Define $V_{f}^{-}(X)$ analogously. The definition of $D_{f}$ immediately implies that the functions $f_{X}^{+}$and $f_{X}^{-}$with

$$
f_{X}^{+}(u)=\left\{\begin{aligned}
f(u), & u \in V(G) \backslash V_{f}^{+}(X), \text { and } \\
f(u)+1, & u \in V_{f}^{+}(X)
\end{aligned}\right.
$$

and

$$
f_{X}^{-}(u)=\left\{\begin{aligned}
f(u), & u \in V(G) \backslash V_{f}^{-}(X), \text { and } \\
f(u)-1, & u \in V_{f}^{-}(X)
\end{aligned}\right.
$$

where we identify colors modulo $k$, are both $k$-colorings of $G$.

Now, let $G$ contain a cycle $C: u_{1} u_{2} u_{3} \ldots u_{k} u_{1}$ of length $k$. We assume that $f$ is chosen such that a longest $f$-rainbow path in $C$ is longest possible. By symmetry, we may assume that $u_{1} \ldots u_{\ell}$ is such a longest $f$-rainbow path in $C$ and that $f\left(u_{i}\right)=i$ for $i \in[\ell]$. By (3),$\ell<k$. Let $f\left(u_{k}\right)=j$. The choice of $P$ implies $j \in[\ell]$. Note that $u_{1}, \ldots, u_{j-1} \in V_{f}^{-}\left(\left\{u_{j}\right\}\right)$ and $u_{j+1}, \ldots, u_{\ell} \in V_{f}^{+}\left(\left\{u_{j}\right\}\right)$, which, by (33), implies $u_{1}, \ldots, u_{j-1} \notin V_{f}^{+}\left(\left\{u_{j}\right\}\right)$ and $u_{j+1}, \ldots, u_{\ell} \notin V_{f}^{-}\left(\left\{u_{j}\right\}\right)$. Similarly, by (33), $u_{k} \notin V_{f}^{+}\left(\left\{u_{j}\right\}\right) \cap V_{f}^{-}\left(\left\{u_{j}\right\}\right)$. Now, if $u_{k} \notin V_{f}^{+}\left(\left\{u_{j}\right\}\right)$, then $u_{k} u_{1} \ldots u_{\ell}$ is a $f_{\left\{u_{j}\right\}}^{+}$-rainbow path in $C$, and, if $u_{k} \notin V_{f}^{-}\left(\left\{u_{j}\right\}\right)$, then $u_{k} u_{1} \ldots u_{\ell}$ is a $f_{\left\{u_{j}\right\}}^{-}$-rainbow path in $C$, which contradicts the choice of $f$, and completes the proof.

Proof of Theorem 4: Let $G$ be a graph of chromatic number 3, and let $D$ be an orientation of $G$. In view of the desired statement, we may assume that $G$ is connected. Since every orientation of a triangle contains a directed path of order 3, we may assume that $G$ is triangle-free. Let $V^{+}=\left\{u \in V(G): d_{D}^{-}(u)=0\right\}$ and $V^{-}=\left\{u \in V(G): d_{D}^{+}(u)=0\right\}$ be the sets of source vertices and sink vertices of $D$, respectively. Since $V^{+}$and $V^{-}$are independent sets of vertices and $G$ is not bipartite, the set $R=V(G) \backslash\left(V^{+} \cup V^{-}\right)$of vertices $u$ of $G$ with $d_{D}^{+}(u), d_{D}^{-}(u)>0$ is not empty. If $R$ is an independent set of vertices, then the function $f$ with

$$
f(u)= \begin{cases}1, & u \in V^{+} \\ 2, & u \in R, \text { and } \\ 3, & u \in V^{-}\end{cases}
$$

is a 3-coloring of $G$ for which every vertex in $R$ lies on a directed full $f$-rainbow path. Hence, we may assume that $R$ is not independent. Let $f$ be a 3-coloring of $G$. We may assume that for every 
vertex $u$ in $R$, all vertices in $N_{G}(u)=N_{D}^{-}(u) \cup N_{D}^{+}(u)$ have the same color. By symmetry, we may assume that $(u, v)$ is an arc of $D$ such that $u, v \in R, f(u)=1$, and $f(v)=2$. Let $w \in N_{D}^{+}(v)$. The above observations imply that the function $f^{\prime}$ with

$$
f^{\prime}(x)=\left\{\begin{aligned}
f(x), & x \in V(G) \backslash\{u\}, \text { and } \\
3, & x=u
\end{aligned}\right.
$$

is a 3-coloring for which $u v w$ is a directed full $f^{\prime}$-rainbow path, which completes the proof.

Acknowledgment We thank Stéphane Bessy for valuable discussion on this topic.

\section{References}

[1] S. Akbari, F. Khaghanpoor, and S. Moazzeni, Colorful paths in vertex coloring of graphs, preprint.

[2] S. Akbari, V. Liaghat, and A. Nikzad, Colorful paths in vertex coloring of graphs, Electron. J. Combin. 18 (2011) \# P17.

[3] M. Alishahi, A. Taherkhani, and C. Thomassen, Rainbow paths with prescribed ends, Electron. J. Combin. 18 (2011) \# P86.

[4] S. Bessy and N. Bousquet, Colorful paths for 3-chromatic graphs, arXiv 1503.00965v1.

[5] T. Gallai, On directed paths and circuits, in: P. Erdős and G. Katona, Theory of Graphs, 115-118, Tihany, Academic Press, New York, 1968.

[6] C. Lin, Simple proofs of results on paths representing all colors in proper vertex-colorings, Graphs Combin. 23 (2007) 201-203.

[7] B. Roy, Nombre chromatique et plus longs chemins d'un graphe, Rev. Franç. Inform. Rech. Opér. 1 (1967) 129-132.

[8] A. Vince, Star chromatic number, J. Graph Theory 12 (1988) 551-559.

[9] X. Zhu, Circular chromatic number: a survey, Discrete Math. 229 (2001) 371-410. 